\title{
Two-Species Continuum Model for Aeolian Sand Ripples
}

\author{
Rebecca B. Hoyle* \\ Department of Applied Mathematics and Theoretical Physics, Silver Street, Cambridge CB3 9EW, United Kingdom \\ Anita Mehta \\ S. N. Bose National Centre for Basic Sciences, Block JD, Sector III Salt Lake, Calcutta 700 091, India
}

(Received 22 July 1999)

\begin{abstract}
We formulate a continuum model for aeolian sand ripples consisting of two species of grains: a lower layer of relatively immobile clusters, with an upper layer of highly mobile grains moving on top. We predict analytically the ripple wavelength, initial ripple growth rate, and threshold saltation flux for ripple formation. Numerical simulations show the evolution of realistic ripple profiles from initial surface roughness via ripple growth and merger.
\end{abstract}

PACS numbers: 81.05.Rm, 05.40.-a, 45.70.Ht, 92.60.Gn

Aeolian sand ripples are formed by the action of the wind on the sand bed in the desert and at the seashore. They have also recently been observed on Mars [1]. Aeolian ripples are a few centimeters in wavelength and their crests lie perpendicular to the prevailing wind direction. Bagnold (1941) made an influential early study [2]. He identified the importance of saltation, where sand grains are entrained by the wind, and whipped along the sand bed, colliding with it at high speed (of order $\leq 1 \mathrm{~m} / \mathrm{s}$ [3]), and causing other grains to jump out of and along the bed, thus sculpting ripples. The impact angle remains roughly constant at about $10^{\circ}-16^{\circ}$ despite the gusting of the wind. The stoss slope of the ripple lies in the range $8^{\circ}-10^{\circ}$ [4]. The lee slope is composed of a short straight section near the crest at an angle of about $30^{\circ}-34^{\circ}$ to the horizontal $[2,4,5]$, followed by a longer and shallower concave section. The deposition of grains on the lee slope of sand dunes leads to oversteepening and avalanching [6,7], which maintains the lee slope at an angle of around $30^{\circ}-$ $34^{\circ}$ near the crest. A similar, if less dramatic, mechanism is likely to hold for ripples.

Numerical simulations of ripples and dunes based on tracking individual sand grains or on cellular automata have in the past few years yielded good qualitative agreement with observations [8]. However, these methods are computationally expensive. Continuum models provide a complementary approach allowing faster calculation of ripple evolution, and the possibility of obtaining certain information, such as the preferred ripple wavelength, analytically. Anderson [9] produced an analytical model of the initial generation of ripples from a flat bed. A one-species analytical continuum model was formulated by Hoyle and Woods [10]. In the current Letter we extend the one-species model [10] to include relaxation effects, inspired by models of sandpiles [11], in order to obtain more realistic ripple profiles and to predict the ripple wavelength and speed. As our work was nearing completion we learned of other work in progress on ripple formation [12] similar in spirit to our own, but with important differences in implementation.
We consider two-dimensional sand ripples: following earlier work on sandpiles [11,13] we assume that the effective surface of the ripple comprises a "bare" surface defined by the local heights of clusters $h(x, t)$ sheathed by a thin layer of flowing mobile grains whose local density is $\rho(x, t)$, with $x$ the horizontal coordinate, and $t$ time. The ripple evolves under the influence of two distinct types of mechanisms. First, the impact of a constant flux of saltating grains knocks grains out of the bare surface, causing them to hop along the ripple surface and land in the layer of flowing grains. This is the underlying cause of ripple formation. Second, the ripples are subject to intracluster and intercluster granular relaxation mechanisms which result in a smoothing of the surface.

Following [10] we consider grains to be bounced out of the bare surface by a constant incoming saltation flux, which impacts the sand bed at an angle $\beta$ to the horizontal. These hopping or "reptating" [14] grains subsequently land in the flowing layer. The saltating grains are highly energetic and continue in saltation upon rebounding from the sand bed. We assume that the number $N(x, t)$ of sand grains ejected per unit time, per unit length of the sand bed, is proportional to the component of the saltation flux perpendicular to the sand surface, giving

$$
N(x, t)=J \sin (\alpha+\beta)=\frac{J\left(\sin \beta+h_{x} \cos \beta\right)}{\left(1+h_{x}^{2}\right)^{1 / 2}},
$$

where $\alpha=\tan ^{-1}\left(h_{x}\right)$, and $J$ is a positive constant of proportionality. We assume that each sand grain ejected from the surface hops a horizontal distance $a$, with probability $p(a)$, and then lands in the flowing layer. We consider the flight of each sand grain to take place instantaneously, since ripples evolve on a much slower time scale than that of a hop. The hop length distribution $p(a)$ can be measured experimentally $[14,15]$, so we consider it to be given empirically. It is possible that $p(a)$ and hence the mean and variance of hop lengths could depend upon factors such as wind speed. The number $\delta n_{0}(x, t)$ of sand grains leaving the surface between positions $x$ and $x+\delta x$ 
in time $\delta t$, where $\delta x$ and $\delta t$ are infinitesimal, is given by $\delta n_{0}(x, t)=N(x, t) \delta x \delta t$. The change $\delta h$ in the surface height satisfies $\delta x \delta h(x, t)=-a_{p} \delta n_{0}(x, t)$, where $a_{p}$ is the average cross-sectional area of a sand grain. In the limit $\delta t \rightarrow 0$ we find that the contribution to the evolution equation for $h(x, t)$ from hopping alone is

$$
h_{t}=-a_{p} N(x, t)=-a_{p} J \frac{\sin \beta+h_{x} \cos \beta}{\left(1+h_{x}^{2}\right)^{1 / 2}} .
$$

There may be regions on the sand bed which are shielded from the incoming saltation flux by higher relief upwind. In these regions there will be no grains bounced out of the surface, and there will be no contribution to the $h_{t}$ equation from hopping. The number $\delta n_{i}(x, t)$ of sand grains arriving on the layer of flowing grains between positions $x$ and $x+\delta x$ in time $\delta t$ is given by

$$
\delta n_{i}(x, t)=\int_{-\infty}^{+\infty} p(a) N(x-a, t) d a \delta x \delta t .
$$

The change in depth of the flowing layer satisfies $\delta x \delta \rho(x, t)=a_{p} \delta n_{i}(x, t)$, and hence the contribution to the evolution equation for the flowing layer depth from hopping alone is

$$
\rho_{t}=a_{p} J \int_{-\infty}^{+\infty} p(a) \frac{\sin \beta+h_{x}(x-a, t) \cos \beta}{\left[1+h_{x}^{2}(x-a, t)\right]^{1 / 2}} d a .
$$

We incorporate diffusive motion [16] as well as processes governing the transfer between flowing grains and clusters, following [11], leading to the equations

$$
\begin{aligned}
h_{t}= & D_{h} h_{x x}-T(x, t)-a_{p} J \frac{\sin \beta+h_{x} \cos \beta}{\left(1+h_{x}^{2}\right)^{1 / 2}}, \\
\rho_{t}= & D_{\rho} \rho_{x x}+\chi\left(\rho h_{x}\right)_{x}+T(x, t) \\
& +a_{p} J \int_{-\infty}^{+\infty} p(a) \frac{\sin \beta+h_{x}(x-a, t) \cos \beta}{\left[1+h_{x}^{2}(x-a, t)\right]^{1 / 2}} d a,
\end{aligned}
$$

where $D_{h}, D_{\rho}$, and $\chi$ are positive constants and where $T(x, t)$, which represents the transfer terms, is given by

$$
T(x, t)=-\kappa \rho h_{x x}+\lambda \rho\left(\left|h_{x}\right|-\tan \alpha\right),
$$

for $0 \leq\left|h_{x}\right| \leq \tan \alpha$ and by

$$
T(x, t)=-\kappa \rho h_{x x}+\frac{\nu\left(\left|h_{x}\right|-\tan \alpha\right)}{\left(\tan ^{2} \gamma-h_{x}^{2}\right)^{1 / 2}},
$$

for $\tan \alpha \leq\left|h_{x}\right|<\tan \gamma$, with $\kappa, \lambda$, and $\nu$ also positive constants. The term $D_{h} h_{x x}$ represents the diffusive rearrangement of clusters while the term $D_{\rho} \rho_{x x}$ represents the diffusion of the flowing grains. The flux-divergence term $\chi\left(\rho h_{x}\right)_{x}$ models the flow of surface grains under gravity. The current of grains is assumed proportional to the number of flowing grains and to their velocity, which in turn is proportional to the local slope to leading order [10]. The $-\kappa \rho h_{x x}$ term represents the inertial filling in of dips and knocking out of bumps on the bare surface caused by rolling grains flowing over the top. The $\lambda \rho\left(\left|h_{x}\right|-\tan \alpha\right)$ term represents the tendency of flowing grains to stick onto the ripple surface at small slopes; it is meant to model the accumulation of slowly flowing grains at an obstacle. Clearly for this to happen the obstacle must be stable, or else it would be knocked off by the oncoming grains, so that this term comes into play only for slopes less than $\tan \alpha$, where $\alpha$ is the angle of repose. The term $\nu\left(\left|h_{x}\right|-\tan \alpha\right)\left(\tan ^{2} \gamma-h_{x}^{2}\right)^{-1 / 2}$ represents tilt and avalanching; it comes into play only for slopes greater than $\tan \alpha$ and models the tendency of erstwhile stable clusters to shed grains into the flowing layer when tilted. This shedding of grains starts when the surface slope exceeds the angle of repose. For slopes approaching the angle $\gamma$, which is the maximum angle of stability, the rate of tilting out of grains becomes very large: an avalanche occurs. This term, among other things, is a novel representation of the well-known phenomena of bistability and avalanching at the angle of repose [17].

We renormalize the model equations setting $x \rightarrow$ $x_{0} \tilde{x}, \quad t \rightarrow t_{0} \tilde{t}, a \rightarrow x_{0} \tilde{a}, \quad \rho \rightarrow \rho_{0} \tilde{\rho}, h \rightarrow h_{0} \tilde{h}$, where $x_{0}=D_{h} / a_{p} J \cos \beta, \quad t_{0}=D_{h} /\left(a_{p} J \cos \beta\right)^{2}, \quad h_{0}=$ $D_{h} \tan \gamma / a_{p} J \cos \beta, \rho_{0}=a_{p} J \sin \beta / \lambda \tan \alpha$, which gives for $0 \leq h_{x} \leq \tan \alpha / \tan \gamma$

$$
\begin{aligned}
& h_{t}=(1+\hat{\kappa} \rho) h_{x x}-\rho \frac{\tan \beta}{\tan \alpha}\left(\left|h_{x}\right|-\frac{\tan \alpha}{\tan \gamma}\right)-f(x) \\
& \rho_{t}= \frac{h_{0}}{\rho_{0}}\left\{-\hat{\kappa} \rho h_{x x}+\rho \frac{\tan \beta}{\tan \alpha}\left(\left|h_{x}\right|-\frac{\tan \alpha}{\tan \gamma}\right)\right\} \\
&+\frac{h_{0}}{\rho_{0}} \int_{-\infty}^{+\infty} p(a) f(x-a) d a \\
&+\frac{D_{\rho}}{D_{h}} \rho_{x x}+\hat{\chi}\left(\rho h_{x}\right)_{x},
\end{aligned}
$$

and for $\tan \alpha / \tan \gamma \leq h_{x}<1$

$$
h_{t}=(1+\hat{\kappa} \rho) h_{x x}-f(x)-\frac{\hat{\nu}\left(\left|h_{x}\right|-\tan \alpha / \tan \gamma\right)}{\left(1-h_{x}^{2}\right)^{1 / 2}},
$$

$$
\begin{aligned}
\rho_{t}= & \frac{h_{0}}{\rho_{0}}\left\{-\hat{\kappa} \rho h_{x x}+\frac{\hat{\nu}\left(\left|h_{x}\right|-\tan \alpha / \tan \gamma\right)}{\left(1-h_{x}^{2}\right)^{1 / 2}}\right\} \\
& +\frac{h_{0}}{\rho_{0}} \int_{-\infty}^{+\infty} p(a) f(x-a) d a \\
& +\frac{D_{\rho}}{D_{h}} \rho_{x x}+\hat{\chi}\left(\rho h_{x}\right)_{x},
\end{aligned}
$$

where the tildes have been dropped and where

$$
f(x)=\left(h_{x}+\frac{\tan \beta}{\tan \gamma}\right)\left(1+h_{x}^{2} \tan ^{2} \gamma\right)^{-1 / 2},
$$


and $\hat{\kappa}=\kappa \rho_{0} / D_{h}, \hat{\nu}=\nu t_{0} / h_{0}, \hat{\chi}=\chi h_{0} / D_{h}$. Wherever the sand bed is shielded from the saltation flux, the hopping term must be suppressed by removing the term $-f(x)$ in the $h_{t}$ equation.

Close to onset of the instability that gives rise to sand ripples, the slopes of the sand bed will be small, since surface roughness is of small amplitude; hence the regime $0 \leq \tan \alpha / \tan \gamma$ is relevant. There are no shielded regions at early times, since the slope of the bed does not exceed $\tan \beta$. Note that $h_{x}=0, \rho=1$ is a stationary solution of Eqs. (9) and (10). Setting $h=\hat{h} e^{\sigma t+i k x}$ and $\rho=$ $1+\hat{\rho} e^{\sigma t+i k x}$, where $\hat{h} \ll 1$ and $\hat{\rho} \ll 1$ are constants, linearizing, and Taylor-expanding the integrand gives a dispersion relation for $\sigma$ in terms of $k$. The presence of the $\left|h_{x}\right|$ term means that strictly we are considering different solutions for sections of the ripple where $h_{x}>0$ and sections where $h_{x}<0$, but in fact the effect of the $\left|h_{x}\right|$ terms appears only as a contribution $\pm \tan \beta / \tan \alpha$ to the bracket $(1 \pm \tan \beta / \tan \alpha)$, where the $h_{x}>0$ case takes the + sign and $h_{x}<0$ case takes the - sign. Since typically we have $\tan \beta / \tan \alpha \ll 1$, the two solutions will not be very different. One growth rate eigenvalue is given by $\sigma=-h_{0} \tan \beta / \rho_{0} \tan \gamma+O(k)$ and is the rate of relaxation of $\rho$ to its equilibrium value of 1. To $O\left(k^{4}\right)$ the other eigenvalue is

$$
\sigma=\left(\bar{a}-1-\hat{\chi} \rho_{0} / h_{0}\right) k^{2}+i A k^{3}+B k^{4},
$$

where

$$
\begin{aligned}
A= & -\frac{1}{2} \overline{a^{2}}+\frac{\rho_{0}}{h_{0}} \frac{\tan \gamma}{\tan \beta}\left(1+\hat{\chi} \frac{\rho_{0}}{h_{0}}-\bar{a}-\frac{D_{\rho}}{D_{h}}\right) \\
& \times\left(1 \pm \frac{\tan \beta}{\tan \alpha}\right), \\
B= & -\frac{1}{6} \overline{a^{3}}-\frac{1}{2} \overline{a^{2}} \frac{\rho_{0}}{h_{0}} \frac{\tan \gamma}{\tan \beta}\left(1 \pm \frac{\tan \beta}{\tan \alpha}\right) \\
& -\frac{\rho_{0}}{h_{0}} \frac{\tan \gamma}{\tan \beta}\left(\bar{a}-1-\hat{\chi} \frac{\rho_{0}}{h_{0}}+\frac{D_{\rho}}{D_{h}}\right) \\
& \times\left\{\bar{a}+\hat{\kappa}+\frac{\rho_{0} \tan \gamma}{h_{0} \tan \beta}\left(1 \pm \frac{\tan \beta}{\tan \alpha}\right)^{2}\right\},
\end{aligned}
$$

and where $\overline{(\cdot)}$ denotes $\int_{-\infty}^{+\infty}(\cdot) p(a) d a$. We have neglected higher order terms as we are looking for long wave modes where $|k|$ is small, since short waves are damped by the diffusion terms. Sand ripples grow if $\bar{a}>1+\hat{\chi} \rho_{0} / h_{0}$, which is equivalent to requiring that $\bar{a} a_{p} J \cos \beta>D_{h}+$ $\chi a_{p} J \sin \beta / \lambda \tan \alpha$ holds in physical variables, giving a threshold saltation flux intensity for ripple growth. This is in agreement with the threshold found in [10]. Since $B$ is negative $(\beta<\alpha)$, the fastest growing mode has wave number $k^{2}=-\left(\bar{a}-1-\bar{\chi} \rho_{0} / h_{0}\right) / 2 B$ with growth rate $\sigma=-\left(\bar{a}-1-\hat{\chi} \rho_{0} / h_{0}\right)^{2} / 4 B$. The allowed band of wave numbers for growing modes is $0<k^{2}<-(\bar{a}-$ $\left.1-\hat{\chi} \rho_{0} / h_{0}\right) / B$. The wave speed is given by $c=$ $-A k^{2}>0$; it is higher for larger $k^{2}$ which implies that shorter waves move faster, as indeed was seen in the numerical simulations described below. The speed is higher for $h_{x}>0$ than for $h_{x}<0$, leading to wave steepening.

The renormalized model equations (9)-(12) were integrated numerically using compact finite differences [18] with periodic boundary conditions. The $-f(x)$ term in the $h_{t}$ equation was suppressed in shielded regions. We used a normal distribution for the hop lengths with mean $\bar{a}$ and variance $s^{2}$. In the run illustrated, we chose $\bar{a}=3.1$, $s=0.1, D_{\rho} / D_{h}=1.0, h_{0} / \rho_{0}=20.0, \hat{\chi}=0.1, \hat{\nu}=$ $1.0, \hat{\kappa}=0.1, \beta=10^{\circ}, \alpha=30^{\circ}$, and $\gamma=35^{\circ}$. The angles were chosen to agree with observational evidence, the ratio $h_{0} / \rho_{0}$ to ensure a thin layer of flowing grains, and the remaining parameters to allow ripple growth. The output was rescaled back into physical variables using $D_{h}=1.0$ and $\lambda=10.0$. The initial conditions for the dimensionless variables were $h=1.0+0.1 \eta_{h}$, $\rho=0.95+0.1 \eta_{\rho}$, where $\eta_{h}$ and $\eta_{\rho}$ represent random noise generated by random variables on $[0,1)$ in order to model surface roughness. In this case $B=-8.47$ and $A=-5.26$ (taking the minus sign in the brackets), giving a preferred wave number of $k=0.352$, and a wave speed of $c=5.26 k^{2}$. The length of the integration domain was chosen to be 10 times the linearly preferred wavelength.

Figure 1 shows the surface height at time $t=10.0 \Delta t$, where $\Delta t=2.78$. Note the emergence of a preferred wavelength, with wave number $k \approx 0.457$ lying in the permitted band for growing modes predicted by the linear stability analysis and arrived at by a process of ripple merger. The wave speed close to onset was also measured and found to be $c \approx(4.13 \pm 0.39) k^{2}$, which is reasonably close to the predicted value. Ripple merger typically occurs when a small fast ripple catches up and merges with a larger slower ripple (Fig. 2), the leading ripple transferring sand to its pursuer until only the pursuer remains. Occasionally a small ripple emerges from the front of the new merged ripple and runs off ahead. Figure 3 shows the surface height $h$ at time $t=89 \Delta t$, with one shallow and one fully developed ripple. Note the long shallow stoss slopes, and the shorter steeper lee slopes with straight sections near the crests and

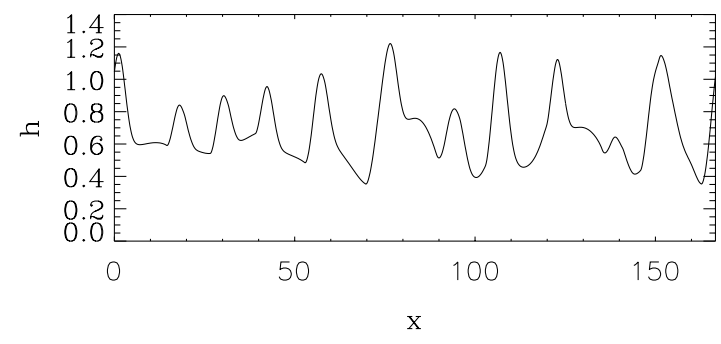

FIG. 1. The surface height $h$ at time $t=10.0 \Delta t$. 


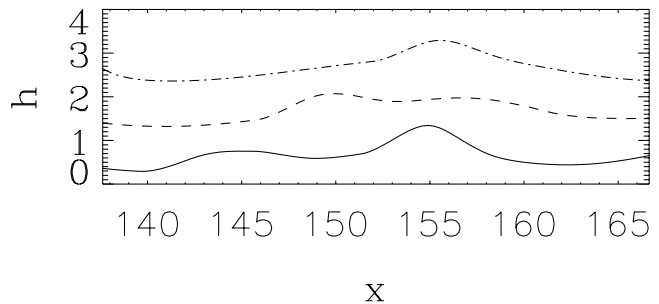

FIG. 2. A sequence of profiles showing a small ripple catching up and merging with a larger ripple. Sand is transferred from the larger to the smaller ripple until only the latter remains. The profiles are shown at times $t=11.0 \Delta t$ (solid line), $t=12.0 \Delta t$ (dashed line), and $t=13.0 \Delta t$ (dot-dashed line). The later profiles are each offset by one additional unit in height.

concave tails. The left-most ripple has a maximum stoss slope angle of $3.3^{\circ}$, and a maximum lee slope of $9.2^{\circ}$, whereas the more fully developed right-most ripple has a maximum stoss slope angle of $24.8^{\circ}$ and a maximum lee slope angle of $33.5^{\circ}$, which lies between the angle of repose and the maximum angle of stability. The height to length ratio of the ripples is in the range 1:8-1:22, which is in reasonable agreement with observations [4]. In the long time limit, we would expect sand ripples to grow until the maximum lee slope angle reaches an angle close to $\tan \gamma$. In reality, there is only a relatively shallow layer of loose sand available for incorportion into ripples, and this together with the maximum slope condition will determine the size of the fully developed ripples.

In summary, we have formulated an analytical continuum model for aeolian sand ripples using a two-species model embodying intracluster and intercluster relaxation, in a description that leads naturally to bistable behavior at the angle of repose, and its cutoff at the maximum angle of stability [17]. We have predicted analytically the preferred ripple wavelength, the wave speed, and the threshold saltation flux required for ripples to form. Our numerical

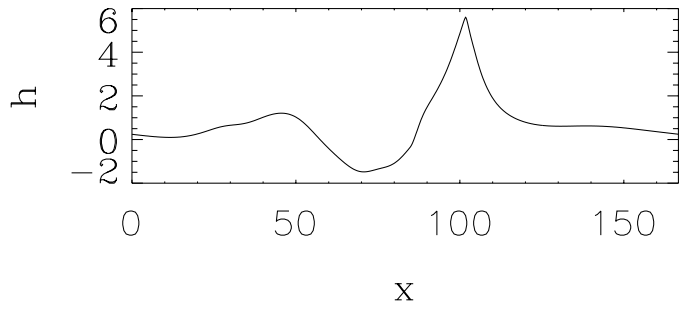

FIG. 3. The surface height $h$ at time $t=89 \Delta t$, showing fully developed ripples. Note the straight segments on the lee slopes close to the crests. simulations show the development of realistic ripple profiles from initial surface roughness via growth and ripple merger.

We thank Neal Hurlburt and Alastair Rucklidge for the use of their compact finite differences code. The work of R. B. H. was supported by King's College, Cambridge. The work of A. M. was supported by EPSRC.

*Email address: R.B.Hoyle@damtp.cam.ac.uk

${ }^{\dagger}$ Email address: anita@boson.bose.res.in

[1] R. Greeley et al., J. Geophys. Res. 104, 8573 (1999).

[2] R. A. Bagnold, The Physics of Blown Sand and Desert Dunes (Methuen and Co., London, 1941).

[3] R.S. Anderson and K. L. Bunas, Nature (London) 365, 740 (1993).

[4] R. P. Sharp, J. Geol. 71, 617 (1963).

[5] K. Pye and H. Tsoar, Aeolian Sand and Sand Dunes (Unwin Hyman, London, 1990).

[6] R. E. Hunter, Sedimentology 32, 409 (1985).

[7] R. S. Anderson, Sedimentology 35, 175 (1988).

[8] R.S. Anderson, Earth-Sci. Rev. 29, 77 (1990); S. B. Forrest and P. K. Haff, Science 255, 1240 (1992); W. Landry and B. T. Werner, Physica (Amsterdam) 77D, 238 (1994); H. Nishimori and N. Ouchi, Int. J. Mod. Phys. B 7, 2025 (1993); Phys. Rev. Lett. 71, 197 (1993); N.B. Ouchi and H. Nishimori, Phys. Rev. E 52, 5877 (1995); H. Nishimori, M. Yamasaki, and K. H. Andersen, Int. J. Mod. Phys. B 12, 257 (1998).

[9] R. S. Anderson, Sedimentology 34, 943 (1987).

[10] R. B. Hoyle and A. W. Woods, Phys. Rev. E 56, 6861 (1997).

[11] A. Mehta, J. M. Luck, and R. J. Needs, Phys. Rev. E 53, 92 (1996); P. Biswas, A. Majumdar, A. Mehta, and J. K. Bhattacharjee, Phys. Rev. E 58, 1266 (1998).

[12] L. Prigozhin, Phys. Rev. E 60, 729 (1999).

[13] A. Mehta, Physica (Amsterdam) 186A, 121 (1992); A. Mehta, in Granular Matter: An Interdisciplinary Approach, edited by A. Mehta (Springer-Verlag, New York, 1994).

[14] S. Mitha, M. Q. Tran, B. T. Werner and P. K. Haff, Acta Mech. 63, 267 (1986).

[15] J. Ungar and P. K. Haff, Sedimentology 32, 267 (1987).

[16] S. F. Edwards and D. R. Wilkinson, Proc. R. Soc. London A 381, 17 (1982).

[17] H. M. Jaeger, S. R. Nagel, and R. P. Behringer, Rev. Mod. Phys. 68, 1259 (1996); A. Mehta and G. C. Barker, Rep. Prog. Phys. 57, 383 (1994); H. M. Jaeger and S. R. Nagel, Science 255, 1523 (1992).

[18] S. K. Lele, J. Comput. Phys. 103, 16 (1992); N. E. Hurlburt and A. M. Rucklidge, Mon. Not. R. Astron. Soc. (to be published). 